\title{
Sucesso após retratamento endodôntico: importância da limpeza e desinfecção do sistema de canais radiculares
}

Success after endodontic retreatment: importance of cleaning and disinfecting the root canal system Éxito después del retratamiento endodóntico: importancia de limpiar y desinfectar el sistema de conducto radicular

Grace Mitiko Rosati HORI

Amanda Albertoni DA SILVA

Marcela POLLAY

David Jonathan Rodrigues GUGSMAN

Anderson CATELAN

Victor Eduardo de Souza BATISTA

Christine Men MARTINS

Faculdade de Odontologia de Presidente Prudente, Universidade do Oeste Paulista (UNOESTE) 19050-920 Presidente Prudente - SP, Brasil

\section{Resumo}

Introdução: A persistência de microrganismos é um dos fatores determinantes de insucesso do tratamento endodôntico, que ocorre devido às iatrogenias, patologias e negligencias. Para solucionar este problema, pode-se indicar a extração dentária ou um tratamento mais conservador, como o retratamento endodôntico. Objetivo: Frente ao exposto, o principal objetivo no presente artigo é relatar um caso clínico de insucesso do tratamento endodôntico primário e o sucesso na abordagem de retratamento que culminou para a finalização positiva do caso clínico. Relato de caso clínico: Paciente com sintomatologia dolorosa ao mastigar no dente 26 foi diagnosticada com periodontite apical crônica com radioluscência periapical por meio da propedêutica clínica e radiográfica com vistas à Endodontia. Foi realizado tratamento endodôntico e após 1 ano da finalização, o paciente apresentava fístula recorrente e foi proposto a extração do dente em questão. Descontente com o plano de tratamento, buscou uma segunda opinião e foi realizado o retratamento endodôntico em sessões múltiplas com a utilização de hidróxido de cálcio como opção para medicação intracanal. Após 60 dias de medicação intracanal, realizou-se a obturação do sistema de canais radiculares e a reabilitação dentária. Conclusão: O sucesso do tratamento endodôntico é dependente da excelente limpeza e desinfecção do sistema de canais radiculares e conseguinte obturação hermética; a persistência de microrganismos é um dos fatores que pode determinar um prognóstico desfavorável e uma nova reintervenção endodôntica pode levar ao sucesso, a despeito de alternativas radicais, como a exodontia e reabilitação com implantes. Por meio do caso clínico apresentado, conclui-se que o planejamento minucioso e a execução do tratamento baseado em evidência científica levam ao sucesso de tratamentos mais conservadores para o paciente e sua satisfatoriedade.

Descritores: Endodontia; Retratamento; Tratamento Conservador; Hidróxido de Cálcio; Periodontite Apical; Fístula.

\section{Abstract}

Introduction: The persistence of microorganisms is one of the determinants of endodontic treatment failure, which occurs due to iatrogenesis, pathologies and negligence. To solve this problem, dental extraction or a more conservative treatment - such as endodontic retreatment, may be indicated. Objective: The aim of this article is to report a clinical case of failure of the primary endodontic treatment and the success in the retreatment approach that culminated in the positive outcome of the clinical case. Clinical case: A patient with painful symptoms on chewing in tooth 26 was diagnosed with chronic apical periodontitis with periapical radiolucency using clinical and radiographic propedeutics for endodontics. Endodontic treatment was performed and 1 year after its completion, the patient had a recurrent fistula and it was proposed to extract the tooth in question. Dissatisfied with the treatment plan, she sought a second opinion and endodontic retreatment was performed in multiple sessions with the use of calcium hydroxide as an option for intracanal therapeutic medication. After 60 days of intracanal medication, filling of the root canal system and dental rehabilitation were performed. Conclusion: The success of the endodontic treatment depends on the excellent cleaning and disinfection of the root canal system and consequently airtight filling; the persistence of microorganisms is one of the factors that can determine an unfavorable prognosis and a new endodontic reintervention can lead to success, despite radical alternatives, such as extraction and rehabilitation with implants. Through the presented clinical case, it is concluded that the meticulous planning and the execution of the treatment based on scientific evidence lead to the success of more conservative treatments for the patient and their satisfaction.

Descriptors: Endodontics; Retreatment; Conservative Treatment; Calcium Hydroxide; Fistula.

\section{Resumen}

Introducción: La persistencia de microorganismos es uno de los determinantes del fracaso del tratamiento endodóntico, que ocurre debido a iatrogénesis, patologías y negligencia. Para resolver este problema, puede estar indicada la extracción dental o un tratamiento más conservador, como el retratamiento endodóntico. Objetivo: En vista de lo anterior, el objetivo principal de este artículo es informar un caso clínico de fracaso del tratamiento endodóntico primario y el éxito en el enfoque de retratamiento que culminó en la conclusión positiva del caso clínico. Informe de caso clínico: Un paciente con síntomas dolorosos al masticar el diente 26 fue diagnosticado con periodontitis apical crónica con radiolucidez periapical utilizando propedéuticos clínicos y radiográficos para endodoncia. Se realizó un tratamiento de endodoncia y 1 año después de la finalización, el paciente tenía una fístula recurrente y se propuso extraer el diente en cuestión. Insatisfecho con el plan de tratamiento, buscó una segunda opinión y el retratamiento endodóntico se realizó en múltiples sesiones con el uso de hidróxido de calcio como una opción para la medicación intracanal. Después de 60 días de medicación intracanal, se llenó el sistema de conducto radicular y se realizó rehabilitación dental. Conclusión: El éxito del tratamiento endodóntico depende de la excelente limpieza y desinfección del sistema de conducto radicular y, en consecuencia, del llenado hermético; la persistencia de microorganismos es uno de los factores que pueden determinar un pronóstico desfavorable y una nueva reintervención endodóntica puede conducir al éxito, a pesar de las alternativas radicales, como la extracción y la rehabilitación con implantes. A través del caso clínico presentado, se concluye que la planificación meticulosa y la ejecución del tratamiento basado en evidencia científica conducen al éxito de tratamientos más conservadores para el paciente y su satisfacción.

Descriptores: Endodoncia; Retratamiento; Tratamiento Conservador; Hidróxido de Calcio; Fístula.

INTRODUÇÃO

O sucesso do tratamento endodôntico está relacionado com a máxima desinfecção do sistema de canais radiculares por meio da ação mecânica dos instrumentos endodônticos, da ação química das substâncias químicas auxiliares, da modelagem e da completa 
obturação com materiais congruentes para que aconteça a cura e reparo dos tecidos periapicais ${ }^{1}$. No entanto, casos de insucesso são relatados na literatura em uma taxa de 2 a $20 \%$, por meio de avaliação radiográfica e dos sinais e sintomas clínicos ${ }^{2}$. A etiologia do insucesso pode estar relacionada com a presença de dor, a persistência de inflamações, infecções e/ou fístulas, a não devolução da estética e função do dente e a ausência de reparo na região periapical ${ }^{1-3}$.

Frente às falhas no tratamento endodôntico primário, algumas opções de tratamento podem estar ao alcance do cirurgiãodentista, como o retratamento endodôntico, a cirurgia paraendodôntica, 0 reimplante intencional, o autotransplante ou a substituição por implantes dentários ${ }^{4}$. A escolha da conduta pelo profissional deve ser baseada na odontologia preventiva e respeitando-se os fatores a serem avaliados, sempre dando preferência para procedimentos menos invasivos $^{4,5}$. Dessa forma, a atenção deve ser voltada para o retratamento endodôntico, embora a literatura mostre índices de sucesso mais baixas quando comparadas as tratamento propriamente dito, especialmente devido às dificuldades técnicas inerentes aos fatores iatrogênicos e eliminação da microbiota resistente ${ }^{6}$.

A literatura indica que a permanência do dente apresenta prognóstico mais favorável a despeito da reabilitação com os implantes dentários devido às complicações, insucesso e doenças como as peri-implantites que não são incomuns, além da taxa de sucesso não ser de $99 \%{ }^{7}$. O tratamento invasivo, associando a exodontia e implantodontia, deve ser de escolha quando outras opções não forem favoráveis e nessa situação são consideradas as vantagens desse tratamento: conforto, estética, ausência de danos aos dentes adjacentes e efeitos clínicos significativos ${ }^{8}$.

Frente ao exposto, o principal objetivo no presente artigo é relatar um caso clinico de insucesso do tratamento endodôntico primário e o sucesso na abordagem de retratamento que culminou para a finalização positiva do caso clinico.

\section{CASO CLÍNICO}

Paciente sexo feminino, 35 anos de idade procurou um cirurgião-dentista relatando dor ao comer. O dente apresentava uma restauração em amálgama extensa e, após a propedêutica clínica e radiográfica, foi diagnosticado periodontite apical crônica com radioluscência periapical no elemento 26 .

Para a realização dessa primeira intervenção endodôntica, foram requeridos 6 meses de tratamento com encontros semanais, devido ao relato da paciente de sintomatologia dolorosa. Apesar do desconforto, o cirurgiãodentista realizou a obturação, com justificativa que não haveria outra solução.

Após 1 ano do procedimento finalizado, a paciente ainda sentia incômodo ao mastigar e culminou com o aparecimento de uma fístula. $\mathrm{Na}$ ocasião a paciente procurou atendimento com outro cirurgião-dentista que afirmou que a lesão poderia ser somente uma afta. O dentista não realizou os procedimentos necessários para a congruência do correto diagnóstico. Nenhum tratamento foi realizado e após alguns dias, houve o desaparecimento da fistula relatada. Porém, dois meses após, a fistula reapareceu, a paciente procurou atendimento com outro profissional que, após a propedêutica clínica e radiográfica, diagnosticou certamente a lesão como uma fístula e observou uma lesão grande no periapice no dente 26 (Figura 1).

Figura 1: Caso clínico inicial. Presença de lesão periapical extensa.

Frente a história clínica do dente em questão, o cirurgião-dentista especialista em Implantodontia propôs o tratamento radical constituído pela exodontia do dente 26, instalação de um implante, seguida da reabilitação com prótese sobre implante. Não satisfeita com essa opção de tratamento, a paciente procurou uma segunda opinião de um especialista em Endodontia. Diferentemente, como um plano de tratamento alternativo e frente ao diagnóstico de insucesso do tratamento endodôntico prévio e à presença da lesão no periapice, foi sugerido o retratamento endodôntico com a consciência da paciente de grandes chances de insucesso devido à probabilidade de trinca/fratura. A despeito dos fatores negativos relativos ao tratamento mais radical, a paciente optou por essa alternativa.

Foi realizada, então, a remoção da restauração insatisfatória e a comprovação que não havia trinca/fratura aparente no soalho da câmara pulpar. Na ocasião, observou-se odor fétido, indicando a presença de infecção. 
Realizou-se a desobturação parcial com a reciproc R40. Observou-se que o instrumento anatômico inicial era correspondente à lima \#70. Foi realizada a reinstrumentação com as limas \#70 e \#80, a agitação das soluções irrigadoras hipoclorito à $2,5 \%$ e a solução de EDTA com o auxílio do dispositivo EasyClean. Após a secagem dos canais, foi introduzida a medicação intracanal de hidróxido de cálcio, iodofórmio e propilenoglicol que permaneceu por 60 dias.

Foi realizado o acompanhamento para a regressão dos sinais e sintomas. Após esse período foi realizada a obturação dos canais pela técnica da condensação lateral com 0 cimento AH Plus e cones de guta percha (Figura 2). Foi realizada a restauração provisória com resina composta e após 4 meses de silêncio clínico o dente foi reabilitado com uma restauração indireta tipo onlay de cerâmica. Houve satisfatoriedade por parte da paciente uma vez que o dente em questão está com sua estética e função devolvidas. Foi realizado o acompanhamento de 24 meses, demonstrando a regressão da lesão (Figura 3).

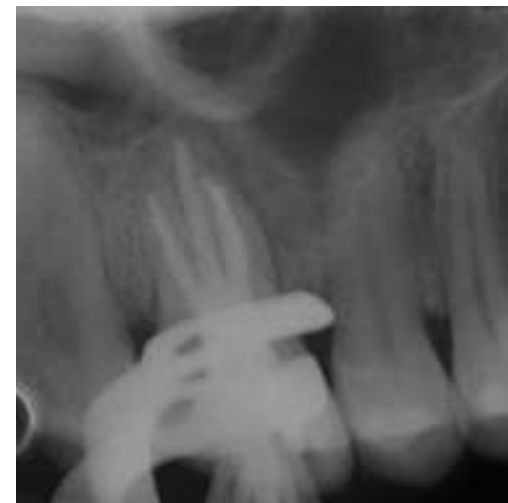

Figura 2: Prova do cone, após 60 dias do uso de medicação intracanal a base de hidróxido de cálcio. Redução da lesão periapical.

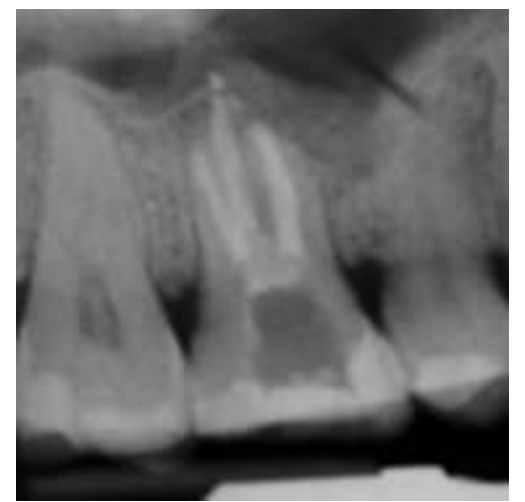

Figura 3: Controle após 24 meses da obturação. Regressão da lesão periapical, silêncio clínico e radiográfico, indicativos de sucesso do retratamento endodôntico.

DISCUSSÃO

O tratamento endodôntico deve ser a escolha em casos em que há diagnóstico que determine o plano de tratamento de necro ou biopulpectomia, sendo considerado conservador quando comparado com à exodontia ${ }^{4}$. No entanto, para haver sucesso frente ao tratamento é necessário o correto diagnóstico, seguido do plano de tratamento e sua execução, reabilitação e a imprescindível proservação do caso ${ }^{2,4}$. Dessa forma, levar-se-á a uma taxa de sucesso entre 80 a $98 \%$.

O insucesso do tratamento está correlacionado à falha no controle asséptico, especialmente ao que tange a remoção de bactérias de canais contaminados e/ou o controle de infecções secundárias ${ }^{1}$. A inabilidade de identificar e acessar áreas infectadas - como a presença de istmos, túbulos dentinários e ramificações do sistema de canais radiculares - influenciam no prognóstico ${ }^{1,9}$.

No caso clínico relatado, a paciente apresentou sintomatologia dolorosa antes e após obturação e presença de fístula recorrente. Esses fatores indicaram insucesso no tratamento realizado. Quando há a persistência de sintomatologia após o procedimento finalizado, a avaliação criteriosa deve ser realizada para identificação da causa do insucesso ${ }^{10}$. A infecção persistente pode gerar uma resposta inflamatória, que está relacionada com a perda óssea e consequentemente falha na cicatrização do tecido, podendo levar à presença de fístulas e dor ${ }^{10}$.

Uma das possibilidades de tratamento relaciona-se com o retratamento endodôntico, dependente da remoção do material obturador presente nos canais, utilizando ou não solventes; reinstrumentação e nova obturação hermética ${ }^{10,11}$.

Outra opção de tratamento é a cirurgia paraendodôntica, que apresenta um índice de sucesso de 66 a $92 \%{ }^{11}$. No entanto essa opção deve ser indicada para casos que somente o retratamento não solucionou, ou frente a presença de canais calcificados, instrumentos fraturados ou pinos intrarradiculares ${ }^{10,11}$.

Com relação à essas duas opções de tratamento, a literatura atual é controversa para entender os efeitos das abordagens cirúrgica versus não cirúrgica e determinar quais procedimentos fornecem os melhores resultados para a cicatrização da lesão periapical e a qualidade de vida pósoperatória ${ }^{10}$.

Atualmente, um dos aspectos mais desafiadores da Odontologia é a escolha entre extração e colocação do implante dentário, a despeito de um tratamento desafiador de canal radicular $^{12}$. A mudança de paradigma e a crescente tendência dos dentistas de substituir o dente pelo implante, em vez do tratamento de 
canal radicular convencional, leva a uma controvérsia $^{12}$, pois muitos dentistas e leigos parecem acreditar que o implante pode oferecer melhores resultados ${ }^{13}$.

De acordo com um estudo prévio ${ }^{14}$, não há diferença em relação a dor e desconforto, taxa de sucesso e de sobrevivência e a qualidade de vida dos pacientes quando comparados o tratamento de canal radicular e a exodontia seguida da instalação de implantes dentários. No entanto, o tempo da reabilitação com implantes dentários pode ser mais longo e com maior custo, além disso os dentes tratados endodonticamente tem mais força mastigatória que os implantes dentários com menor necessidade de tratamento complementar ${ }^{14}$.

No caso clínico apresentado, a paciente bem instruída tem vistas ao tratamento mais conservador. Esse aspecto foi alinhado com o pensamento atual e baseado em evidência científica e notou-se que a opção escolhida foi de sucesso.

O tratamento endodôntico só é finalizado quando o dente apresenta silêncio clínico, características radiográficas congruentes com o processo de cura e reparo e a reabilitação que permite a devolução da estética e função do dente. No caso clínico apresentado, foi realizada inicialmente uma restauração em resina composta para a observância dos dois primeiros aspectos. Confirmado o sucesso do retratamento, foi realizada a restauração indireta tipo onlay como forma definitiva da reabilitação.

As onlays são indicadas para casos de reabilitação ampla em dentes posteriores em preparos parciais que envolvem uma ou mais cúspides. As vantagens dessa opção consistem na facilidade da reprodução dos contatos interproximais e mínima contração de polimerização comparada às resinas diretas ${ }^{15}$.

No entanto, independentemente do tipo de reabilitação e respeitando-se as suas indicações, a literatura é clara ao elucidar a importância do selamento coronal após a endodontia. A sua ausência ou insatisfatoriedade pode levar à recontaminação do sistema de canais radiculares - por meio de infiltrações devido à desadaptação do material restaurador, pericementites devido ao ajuste oclusal inadequado ou mesmo fraturas dentárias - em decorrência da associação da má adaptação da restauração e forças oclusais excessivas que podem gerar fraturas ${ }^{16,17}$.

CONSIDERAÇÕES FINAIS

O sucesso do tratamento endodôntico é dependente de uma adequada limpeza e desinfecção do sistema de canais radiculares e conseguinte obturação hermética; a persistência de microrganismos é um dos fatores que pode determinar um prognóstico desfavorável e uma nova reintervenção endodôntica pode levar ao sucesso, a despeito de alternativas radicais, como a exodontia e reabilitação com implantes. Por meio do caso clínico apresentado, concluise que o planejamento minucioso e a execução do tratamento baseado em evidência científica levam ao sucesso de tratamentos mais conservadores para 0 paciente e sua satisfatoriedade.

REFERÊNCIAS

1. Yamaguchi $M$, Noiri $Y$, Itoh $Y$, Komichi $S$, Yagi $\mathrm{K}$, Uemura $\mathrm{R}$ et al. Factors that cause endodontic failures in general practices in Japan. BMC Oral Health. 2018;18(1):70.

2. Prada I, Micó-Muñoz P, Giner-Lluesma T, MicóMartínez P, Collado-Castellano N, ManzanoSaiz A. Influence of microbiology on endodontic failure. Literature review. Med Oral Patol Oral Cir Bucal. 2019;24(3):e364-72.

3. Yücel O, Ekici MA, Ilk O, Ilhan MN, Kayaoglu G. Predicting intraoperative pain in emergency endodontic patients: clinical study. Braz Oral Res. 2018;32:e38.

4. Torabinejad M, White SN. Endodontic treatment options after unsuccessful initial root canal treatment: Alternatives to single-tooth implants. J Am Dent Assoc. 2016;147(3):214-20.

5. Chatzopoulos GS, Koidou VP, Lunos S, Wolff LF. Implant and root canal treatment: Survival rates and factors associated with treatment outcome. J Dent. 2018;71:61-6.

6. Santos-Junior AO, De Castro Pinto L, MateoCastillo JF, Pinheiro CR. Success or failure of endodontic treatments: A retrospective study. J Conserv Dent. 2019;22(2):129-32.

7. Clark D, Levin L. In the dental implant era, why do we still bother saving teeth? Dent Traumatol. 2019;35(6):368-75.

8. Sebring $D$, Kvist $T$, Derks J. Indications for Extraction before Implant Therapy: Focus on Endodontic Status. J Endod.2019;45(5):532-37.

9. Lacerda MFLS, Coutinho TM, Barrocas D, Rodrigues JT, Vidal F. Infecção secundária e persistente e sua relação com o fracasso do tratamento endodôntico. Rev Brasileira de Odontol. 2016;73(3):212-17.

10.Del Fabbro M, Corbella S, Sequeira-Byron P, Tsesis I, Rosen E, Lolato A et al. Endodontic procedures for retreatment of periapical lesions. Cochrane Database Syst Rev. 2016;10: CD005511.

11. Kang $M$, In Jung $H$, Song $M$, Kim SY, Kim HC, Kim E. Outcome of nonsurgical retreatment and endodontic microsurgery: a meta-analysis. Clin Oral Investig. 2015;19(3):569-82. 
12. Di Fiore PM, Tam L, Thai HT, Hittelman E, Norman RG. Retention of teeth versus extraction and implant placement: treatment preferences of dental faculty and dental students. J Dent Educ. 2008;72(3):352-58.

13. Setzer FC, Kim S. Comparison of long-term survival of implants and endodontically treated teeth. J Dent Res. 2014;93(1):19-26

14. Parirokh M, Zarifian A, Ghoddusi J. Choice of Treatment Plan Based on Root Canal Therapy versus Extraction and Implant Placement: A Mini Review. Iran Endod J. 2015;10(3):152-55.

15. Angeletaki F, Gkogkos A, Papazoglou E, Kloukos D. Direct versus indirect inlay/onlay composite restorations in posterior teeth. A systematic review and meta-analysis. J Dent. 2016;53:12-21.

16. Pratt I, Aminoshariae A, Montagnese TA, Williams KA, Khalighinejad N, Mickel A. EightYear Retrospective Study of the Critical Time Lapse between Root Canal Completion and Crown Placement: Its Influence on the Survival of Endodontically Treated Teeth. J Endod. 2016;42(11):1598-603.

17. Stenhagen $S$, Skeie $H$, Bårdsen $A$, Laegreid $T$. Influence of the coronal restoration on the outcome of endodontically treated teeth. Acta Odontol Scand. 2020;78(2):81-6.

\section{CONFLITO DE INTERESSES}

Os autores declaram não haver conflitos de interesse

\section{AUTOR PARA CORRESPONDÊNCIA}

\section{Christine Men Martins}

Faculdade de Odontologia

Universidade do Oeste Paulista (UNOESTE),

Rua José Bongiovani, 700 - Cidade Universitária 19050-920 Presidente Prudente - SP, Brasil

Tel. +55 (18) $3229-2003$

e-mail: christinemen@hotmail.com 